\title{
Design improvement of the target elements of Wendelstein 7-X divertor
}

\author{
J. Boscary ${ }^{\mathrm{a}}$, A. Peacock ${ }^{\mathrm{c}}$, T. Friedrich ${ }^{\mathrm{b}}$, H. Greuner ${ }^{\mathrm{a}}$, B. Böswirth ${ }^{\mathrm{a}}$, H. Tittes ${ }^{\mathrm{a}}$, \\ W. Schulmeyer ${ }^{\mathrm{b}}$, F. Hurd ${ }^{\mathrm{c}}$ \\ ${ }^{a}$ Max-Planck-Institut für Plasmaphysik, EURATOM Association, 85748 Garching, Germany \\ ${ }^{b}$ Plansee SE, Innovation Services, 6600 Reutte, Austria \\ ${ }^{c}$ European Commission c/o Max-Planck-Institut für Plasmaphysik, EURATOM Association, 85748 Garching, Germany
}

\begin{abstract}
The actively cooled high-heat flux divertor of the Wendelstein 7-X stellarator consists of individual target elements made of a water-cooled CuCrZr copper alloy heat sink armoured with CFC tiles. The so-called "bi-layer" technology developed in collaboration with the company Plansee for the bonding of the tiles onto the heat sink has reliably demonstrated the removal of the specified heat load of $10 \mathrm{MW} / \mathrm{m}^{2}$ in the central area of the divertor. However, due to geometrical constraints, the loading performance at the ends of the elements is reduced compared to the central part. Design modifications compatible with industrial processes have been made to improve the cooling capabilities at this location. These changes have been validated during test campaigns of full-scale prototypes carried out in the neutral beam test facility GLADIS. The tested solution can remove reliably the stationary heat load of $5 \mathrm{MW} / \mathrm{m}^{2}$ and $2 \mathrm{MW} / \mathrm{m}^{2}$ on the top and on the side of the element, respectively. The results of the testing allowed the release of the design and fabrication processes for the next manufacturing phase of the target elements.
\end{abstract}

Keywords: Stellarator, Plasma Facing Components, Divertor

\section{Introduction}

The actively cooled high-heat flux (HHF) divertor of stellarator Wendelstein 7-X (W7-X) is a key component for the successful high power steady state operation of this machine. W7-X is designed for steady-state operation with $10 \mathrm{MW}$ input power over a pulse length of up to 30 minutes and peak power of up to $20 \mathrm{MW}$ for 10 seconds [1].

The design requirements of the divertor components are based on defined standard magnetic configurations where the strike points load the central part of the target elements with a specified stationary heat load of 10 $\mathrm{MW} / \mathrm{m}^{2}$ [2]. However, due to the water cooling geometry of the elements, the loading performance at the end of the target surface is reduced compared to the central part. Design modifications have been investigated to improve the cooling capabilities at this location. Two aspects of the design of the target elements have been considered: the design of the cooling structure and the arrangement of the tiles armouring at the end of the element. As with the central part of the target elements, the assessment of the performance is based on HHF testing of full-scale prototypes in the GLADIS facility [3].

\section{Target elements}

\subsection{Divertor Design}

The divertor consists of ten similar discrete units, two units per period. An open configuration was selected to achieve effective power and particle exhaust over a wide range of magnetic configurations and plasma parameters. As shown in Fig. 1, the target surface of the divertor installed in the plasma vessel is split into the vertical part mounted at the inner side of the vessel and the horizontal part mounted at the outer side of the vessel. The horizontal (part 1) and vertical target plates define the main pumping gap. Each divertor unit consists of 10 HHF target modules, 100 modules for the whole divertor. Each module is a set of 8-12 target elements mounted onto a support frame and fed in parallel from water manifolds.

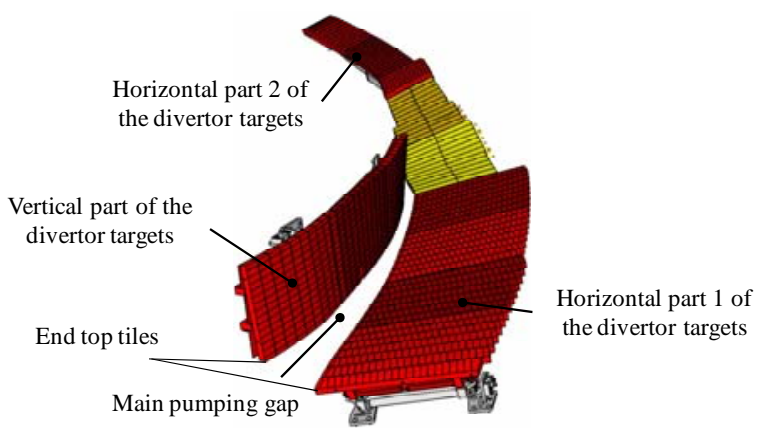

Fig.1. Overview of one divertor unit (without piping).

The positioning of the main pumping gap requires the installation of the piping at the opposite extremity of the elements to avoid thermal loading of the water connectors.

\subsection{Target element design \& technology}

In total there are 890 target elements of twelve different types in total ranging from 250 to $600 \mathrm{~mm}$ length and 50 to $61.5 \mathrm{~mm}$ width. The shape of the elements is slightly trapezoidal along and across the element. The multiplicity of types is mainly due to 
diagnostics integration. The design is based on the flat tiles made from CFC NB31 joined to a CuCrZr heat sink. The $19 \mathrm{~m}^{2} \mathrm{HHF}$ divertor surface consists of 18,000 tiles.

The manufacturer Plansee (Reutte, Austria) produces the elements in two phases. The first phase comprises the shorter and simpler elements used in the horizontal part 2 of the divertor (see Fig. 1). The serial fabrication started in 2009 after an extensive collaborative validation phase [4]. One of the main achievements is the selection of the so-called "bi-layer" technology for the bonding of the CFC tiles onto the heat sink [5, 6]. The delivery of these elements is completed.

The second phase is the fabrication of the elements facing the main pumping gap. These elements are longer and armoured at the end. The technology for these additional features was validated by the fabrication of full-scale prototype elements which were inspected and HHF tested in the GLADIS facility. The manufactured elements, delivered by Plansee in 2011, are shown in Fig. 2. The length of the elements placed in the centre is $600 \mathrm{~mm}$ and on the side $361 \mathrm{~mm}$. The elements are equipped with special water connections for testing.

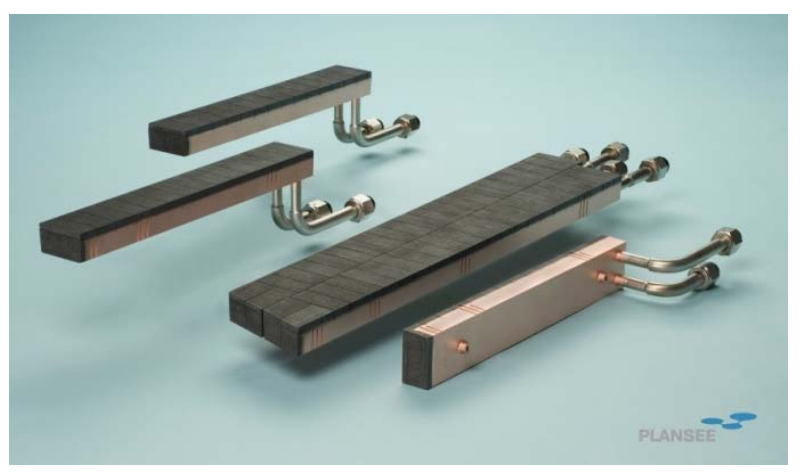

Fig.2 Target element prototypes (Source: Plansee)

\subsection{Design improvements of the cooling structure}

Fig. 3 illustrates the limited cooling at the end of the element due to the design for the element in the case of the horizontal divertor. It shows the end of the element from the back side and the design of the cooling channel inside the cooling structure.

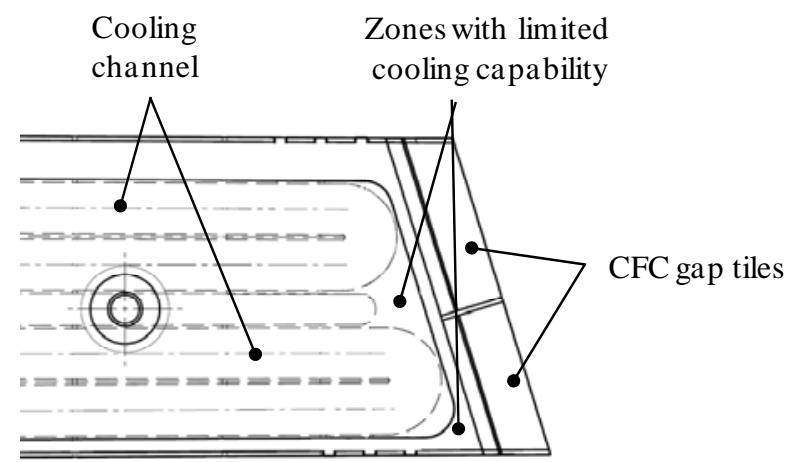

Fig.3. Detail of the cooling channel design at the end of the element
In the central part behind the CFC gap tiles (see Fig. 5), the cooling capability is intrinsically restricted due to the geometry of the element.

This makes it particularly important to ensure adequate water flow to this area. The cooling structure is made of a body closed with a back plate both made of $\mathrm{CuCrZr}$ joined together by electron beam welding. Semi circular channels, $9 \mathrm{~mm}$ in diameter, are machined into each of the two half plates. A swirl tape, $1 \mathrm{~mm}$ thick stainless steel, of ratio 2 is placed in the straight parts of the cooling channel, mechanically attached to the heat sink by a key-slot system. The thickness of the target element shown in Fig. 4 is $30 \mathrm{~mm}$.

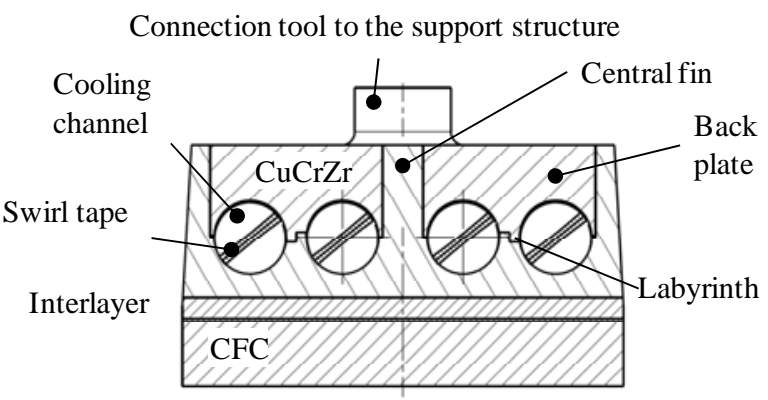

Fig.4 Cross section of the target element

During the pre-series phase, the impact of water leakage between the adjacent channels of the cooling structure on the performance and life time of the elements was investigated [7]. The consequent reduction of the local water velocity at the end of the element reduced the local heat transfer and caused an increase in temperature. This effect is particularly significant for the longest $(600 \mathrm{~mm})$ elements. Therefore, as shown in Fig. 3, a sealing labyrinth has been machined between two adjacent channels on each side of the element.

\subsection{Design improvement: end of horizontal divertor}

The elements of the horizontal divertor (part 1 in Fig. 1) have an angle of $17^{\circ}$ resulting from the divertor geometry. Fig. 5 shows the evolution of the design.

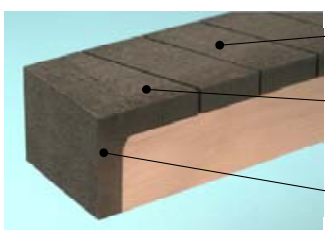

First design
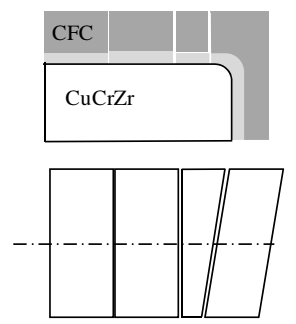

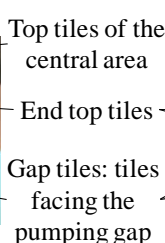

pumping ga

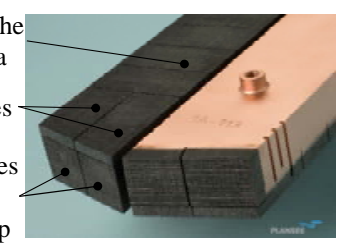

New design
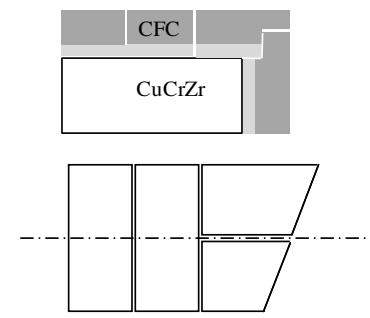

Fig.5. Design of the end tiles of the horizontal divertor 
In the first design an L-shaped tile protects the side facing the pumping gap and the top end. A triangular tile is shaped to make the transition to the top tiles of the central area. This geometry had the advantage of offering a continuous welded transition between the side and the top parts, at a location where the cooling is less efficient due to the distance to the channel. The disadvantage of this design was the high consumption of CFC blocks used for the L-shaped tiles. HHF testing performed in GLADIS in 2005 always showed failure of the triangular-shaped tile due to its geometry but the Lshaped tile withstood the specification [8]. The new arrangement of the tiles is a combination of flat tiles only and allows a better standardization of the fabrication of the tiles. Two tiles, almost square, are positioned on the side (gap tiles) and two trapezoidal top tiles with a $90^{\circ}$ rotation with respect to the other top tiles are attached on the top. The disadvantage of this design is the un-cooled overlap located at the end of the top tile.

\subsection{Design improvement: end of vertical divertor}

The full-scale prototypes of the vertical divertor elements are shown in Fig. 6. The end of the elements does not require a special shaping to fit the main pumping gap, which allowed for a more simplified design using flat tiles.

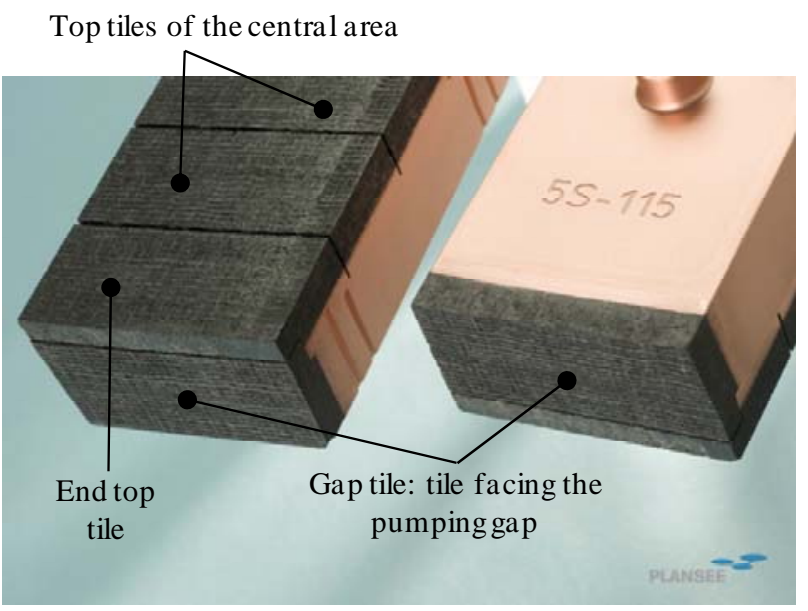

Fig.6. Design of the end tiles of the vertical divertor

\section{Testing in GLADIS}

In operation the top surface of the element will be loaded with convective power whereas the side facing the pumping gap will be loaded with mainly radiation and neutral particles fluxes. For the validation of the design and technology the full-scale prototypes are tested in GLADIS. The end of the top part of the element is designed to withstand a heat flux of 5 $\mathrm{MW} / \mathrm{m}^{2}$. The side facing the pumping gap is designed for $2 \mathrm{MW} / \mathrm{m}^{2}$. The validation is based on the results obtained after 100 thermal cycles. The water cooling conditions are: the inlet temperature is room temperature, the axial velocity is $8 \mathrm{~m} / \mathrm{s}$, the outlet pressure is $1 \mathrm{MPa}$.
The main purpose of the HHF testing is to investigate the performance of the interface between the CFC and the heat sink. It has to be demonstrated that no deterioration of the performance of the element occurs during thermal cycling, under the specified loads and cooling conditions. In total 6 full-scale prototypes were tested: 3 for the geometry of the horizontal divertor and 3 for the vertical one.

The campaign was divided into two stages: the testing of the top tiles, and the testing of the combination of the end top and gap tiles. For the first stage the surface of the element faces directly the beam. The beam centre position and the beam parameters are adjusted to load the end top tiles with a heat flux of 5 $\mathrm{MW} / \mathrm{m}^{2}$. For the second stage the element is positioned with an angle of $45^{\circ}$ with respect to the beam axis. The beam parameters are adjusted to load the end of gap tile with $2 \mathrm{MW} / \mathrm{m}^{2}$. The beam centre is positioned at $70 \mathrm{~mm}$ from the end of the element. This arrangement is shown in Fig. 7.

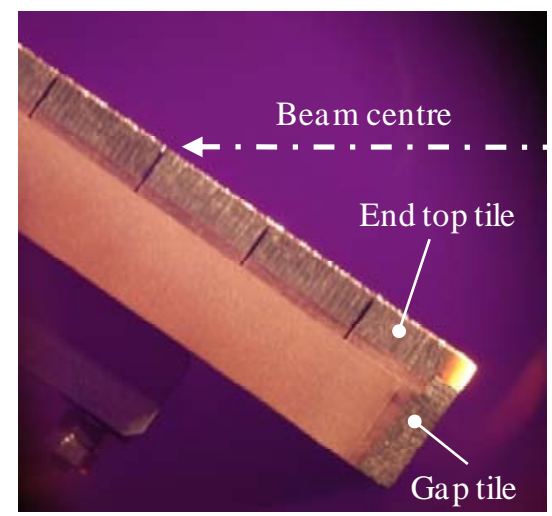

Fig.7. HHF testing of end tile of the vertical divertor

Fig. 7 shows the picture of the end of the elements during HHF testing taken with a CCD camera. The end of the top tile has the lowest cooling capability.

The analysis of the experimental results after 100 thermal cycles showed no deterioration of the bond at the interface between CFC and heat sink and no degradation of the heat transfer at the end of the element. No cracks or crack initiation at the interface between tiles and heat sink have been detected during the subsequent detailed visual inspection.

In a following step and for the second stage the input power was increased to probe the margin of the element. The input power was increased stepwise up to the detection of a stable hot spot with the infrared camera and a significant surface temperature increase. Fig. 8 presents the evolution of the average temperature measured with the infrared camera as a function of the incident heat flux. It is measured at the side of the end top tile. The maximum heat flux of $7.5 \mathrm{MW} / \mathrm{m}^{2}$ corresponds to a CFC surface temperature of about $2000^{\circ} \mathrm{C}$. At this level a partial melting of the interlayer was detected by the infrared camera. 


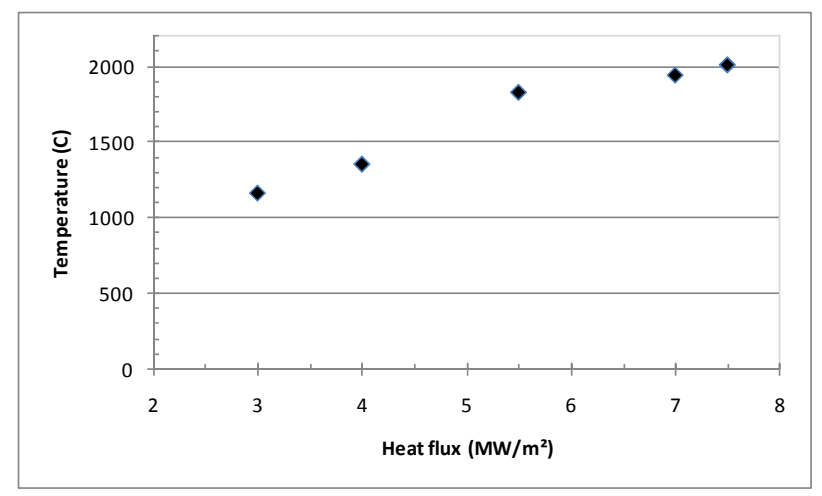

Fig.8. Temperature evolution at the side of the end top tile

The partial de-bonding of the tile was confirmed by the visual inspection carried out afterwards, as shown in Fig. 9. The de-bonding is located at the end of the top tile with a length of about $3 \mathrm{~mm}$ across the tile.

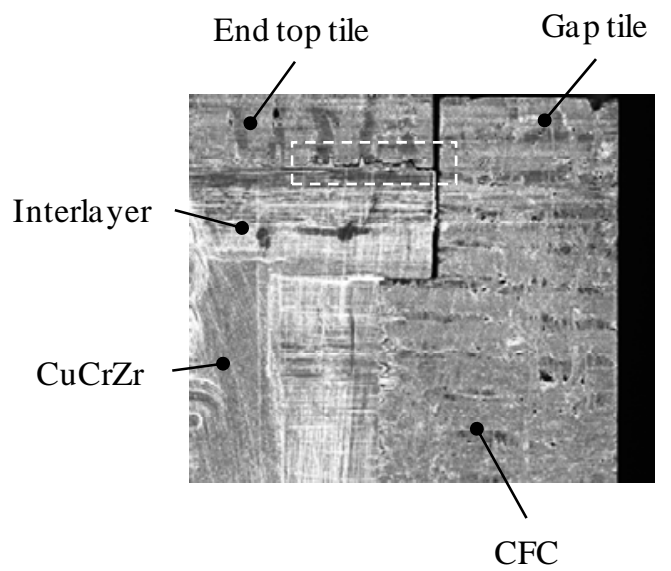

Fig.9. De-bonding of the end top tile for the vertical divertor after HHF testing

These test results demonstrate the limit and the margin of the design. The end top tile defines the power limits of the end of the target elements, both for the vertical or the horizontal divertor. The limitation does not come from the gap tile, which was able to withstand the same magnitude of $7.5 \mathrm{MW} / \mathrm{m}^{2}$, and no de-bonding or crack was detected at the interface. At this level the damage of the end is initiated and also detectable.

\section{Conclusion}

The design of the W7-X HHF target elements has been modified to improve the performances of the ends by the introduction of a sealing labyrinth between adjacent channels of the cooling structure and new arrangement of the tiles protecting the top end and the edge facing the main pumping gap for the horizontal and vertical parts of the divertor. These modifications have been validated by the production of full-scale prototypes and by HHF testing of these prototypes in the neutral beam GLADIS facility. It has been demonstrated that the design of the end tiles can reliably remove under stationary conditions $5 \mathrm{MW} / \mathrm{m}^{2}$ and $2 \mathrm{MW} / \mathrm{m}^{2}$ on the top and on the edge of the element, respectively. The results of this experimental campaign allowed the release of the design and fabrication processes for the complete manufacturing of the target elements. The limits of the design have also been investigated. The degradation of the end of the element starts at a heat flux of 7.5 $\mathrm{MW} / \mathrm{m}^{2}$, associated with a high CFC surface temperature. Further work is needed to assess the performance of the elements installed onto the target modules requiring a final shaping of the element ends with a reduction in the CFC thickness and the life time with a higher number of thermal cycling. These experimental results should be carefully analyzed because recent plasma scenarios taking bootstrap current effects into account have shown the possible drift of the strike points over the end of the target elements of the divertor [9].

\section{Acknowledgments}

This work has been performed under the grant $\mathrm{Nr}$. 03FUS0014A from the Federal Ministry of Education and Research. Only the authors are responsible for the content of this publication.

\section{References}

[1] H. Renner et al., The capabilities of steady state operation at the stellarator W7-X with emphasis on divertor design, Nuclear Fusion 40 (6) (2000) 1083-1093.

[2] J. Kisslinger et al., Proceedings of the 21st EPS Conference on Controlled Fusion and Plasma Physics, Montpellier 1994, ECA 18B European Physics Society, Geneva, Part I (1994) 368.

[3] H. Greuner et al., Design, performance and construction of a 2 MW ion beam test facility for plasma facing components, Fusion Engineering and Design 75-79 (2005) 345-350.

[4] J. Boscary et al., Pre-series and testing route for the serial fabrication of W7-X target elements, Fusion Engineering and Design 84 (2009) 497-500.

[5] H. Greuner et al., Review of the high heat flux testing as an integrated part of W7-X divertor development, Fusion Engineering and Design (2009) 848-852.

[6] A. Plankensteiner et al., Finite element based design optimization of Wendelstein 7-X divertor components under high heat flux loading, Fusion Engineering and Design 82 (2007) 1813-1819.

[7] M. Smirnow et al., Development of a thermo-hydraulic bypass leakage test method for the Wendelstein 7-X target element cooling structure, Fusion Engineering and Design in Press.

[8] H. Greuner et al., High heat flux facility GLADIS: Operational characteristics and results of W7-X pre-series target tests Journal of Nuclear Materials, 367-370, Part 2, (2007), 1444-1448.

[9] J. Geiger et al., Physics Modeling for Steady-State Experiments at Wendelstein 7-X, 23rd IAEA Fusion Energy Conf., THC-P2-02 (2010), in Press. 